\title{
Continuous topical heat was as effective as ibuprofen for dysmenorrhoea
}

\author{
Akin MD, Weingand KW,Hengehold DA, et al. Continuous low-level topical heat in the treatment of dysmenorrhea. Obstet \\ Gynecol 2001 Mar;97:343-9.
}

\section{QUESTION: Is continuous, low level, topical heat as effective as oral ibuprofen for dysmenorrhoea (menstrual pain)?}

\section{Design}

Randomised \{allocation concealed\}*, blinded \{patients, clinicians, and outcome assessors $\}^{*}$, controlled, $2 \times 2$ factorial trial with follow up over 2 days of treatment.

\section{Setting}

Texas, USA

\section{Patients}

84 non-pregnant women who were $\geqslant 18$ years (age range $21-50$ y) and who predictably had moderate or greater menstrual pain (4 of previous 6 cycles), a history and physical examination consistent with primary dysmenorrhoea, regular menstrual cycles, pain relief from over the counter analgesics, and were using reliable contraception. Exclusion criteria were cutaneous lesions involving the abdominal wall, microvascular disease (including diabetes), known or suspected drug or alcohol abuse, known or suspected contraindication to oral ibuprofen, and recent pregnancy. 79 women (94\%) were included in the efficacy analysis.

\section{Intervention}

Women were allocated to 1 of 4 treatment groups, each of which comprised a combination of a heated or unheated patch and ibuprofen or placebo. Women were asked to wear a kidney bean shaped, ultra thin, heated or unheated patch that adhered to the inside of the underwear on the lower abdominal region for 12 consecutive hours each day for 2 consecutive days and to take 2 tablets of oral medication (ibuprofen, $200 \mathrm{mg}$ or placebo) 3 times each day approximately 6 hours apart. 20 women were allocated to a heated patch plus ibuprofen (heat plus ibuprofen), 20 to a heated patch plus placebo (heat alone), 21 to an unheated patch plus ibuprofen (ibuprofen alone), and 20 to an unheated patch plus placebo (control).

\section{Main outcome measures}

Weighted mean changes in baseline pain relief ( 6 point scale, $0=$ no relief to $5=$ complete relief) and pain intensity (101 point numerical rating scale, $0=$ no pain to $100=$ worst possible pain) scores over the 2 study days.

\section{Main results}

Over the 2 study days, women in the heat plus ibuprofen $(\mathrm{p}<0.001)$, heat alone $(\mathrm{p}<0.001)$, and ibuprofen alone $(\mathrm{p}=0.001)$ groups had greater pain relief than women in the control group. Heat plus ibuprofen did not differ from ibuprofen alone for pain relief $(p=0.096)$; however, heat plus ibuprofen resulted in faster improvement in pain relief (median $1.5 v 2.79 \mathrm{~h}$ to onset of noticeable pain relief, $p=0.01$ ). More women in the heat plus ibuprofen and the heat alone groups had complete pain relief compared with women in the control group (table).
The mean baseline pain intensity score was 69.2 out of 100 points. Over the 2 study days, mean reduction in pain intensity score was greater for the heat plus ibuprofen (43.8), heat alone (40.4), and ibuprofen alone (39.0) groups compared with the control group (21.9) $(\mathrm{p}<0.003)$; the heat alone group did not differ from the ibuprofen alone group $(\mathrm{p}=0.8)$, and the heat plus ibuprofen group did not differ from the ibuprofen alone group $(\mathrm{p}=0.22)$.

\section{Conclusion}

Continuous, low level topical heat was as effective as ibuprofen for dysmenorrhoea.

*Information provided by author.
Source of funding: Procter and Gamble Company supplied study devices and materials.

For correspondence: Dr R P Smith,

Department of Obstetrics Medical Center, 2301

Holmes Street, Kansas City, $M O$ 64108, USA. Fax +18165563556 and Gynecology, Truman

Heat plus ibuprofen and heat alone $v$ no heat plus placebo (control) for complete pain relief over 2 study dayst

\begin{tabular}{lrrr} 
Comparison & Event rates & RBI (95\% Cl) & NNT (Cl) \\
Heat + ibuprofen $v$ control & $68 \% v 35 \%$ & $94 \%(4$ to 289$)$ & 3 (2 to 28$)$ \\
\hline Heat alone $v$ control & $70 \% v 35 \%$ & $100 \%(9$ to 298$)$ & 3 (2 to 18$)$ \\
\hline
\end{tabular}

†Abbreviations defined in glossary; RBI, NNT, and $\mathrm{Cl}$ calculated from data in article.

\section{COMMENTARY}

Limited experimental research has been done on the non-pharmacological treatment of dysmenorrhoea. A recent retrospective examination of adolescents' management of menstrual discomfort showed that $98 \%$ of 289 girls reported using $\geqslant 1$ nonpharmacological method to control pain. ${ }^{1}$ The study by Akin $e t$ al is one of the few studies that have attempted an experimental comparison of a non-pharmacological method (topically applied heat) with oral ibuprofen and placebo for menstrual pain.

The effort to investigate topically applied heat to control menstrual pain is a logical endeavour, given the common use of heating pads and hot water bottles to treat menstrual discomfort. Additionally, topical heat is commonly applied to treat other types of inflammatory discomfort. Many nurses recommend topical heat application for musculoskeletal trauma, arthritic discomforts, and other painful ailments. A recent study showed arthritic pain relief with the application of warm mud packs, which caused a decrease of serum prostaglandin and leukotriene levels. ${ }^{2}$

The study by Akin $e t$ al does not provide a cost effectiveness analysis on the use of thermal patches to treat dysmenorrhoea, but the treatment appears to be clinically effective given the results of this small, randomised, placebo controlled trial. The results suggest a useful alternative for those patients who prefer to manage pain using non-pharmacological methods.

Dysmenorrhoea is a painful and sometimes debilitating disorder that can adversely affect the quality of life of many women for several days per month during their child bearing years. The results of this study provide a useful treatment alternative that nurses who work with women experiencing menstrual pain may suggest. The application of a small, thermal patch placed inside a woman's underwear may allow her to continue her daily routine while obtaining relief without the use of drugs.

Cathy R Kessenich, RN, DSN, ARNP Associate Professor of Nursing/Nurse Practitioner University of Tampa Tampa, Florida, USA

1 Campbell MA, McGrath PJ. Non-pharmacologic strategies used by adolescents for the management of menstrual discomfort. Clin J Pain 1999;15:313-20.

2 Bellometti S, Galzigna L. Serum levels of a prostaglandin and a leukotriene after thermal mud pack therapy. J Investig Med 1998;46:140-5. 